\title{
Reentrant spin-glass behavior induced by the frustration of Fe-Fe interactions in Laves phase $\mathrm{Nb}_{1-x} \mathrm{Hf}_{x} \mathrm{Fe}_{2}$ alloys
}

\author{
Tian Gao, ${ }^{1,2, \dagger}$ Katsuhiko Nishimura, ${ }^{2}$ Takahiro Namiki, ${ }^{2}$ and Hiroshi Okimoto ${ }^{2}$ \\ ${ }^{1}$ Department of Physics, Shanghai University of Electric Power, Shanghai 201300, China \\ ${ }^{2}$ Graduate School of Science and Engineering, University of Toyama, Toyama 930-8555, Japan \\ † Corresponding author, E-mail: gaotianok@gmail.com
}

\section{Abstract}

We carried out magnetization and specific-heat measurements of metallic compounds $\mathrm{Nb}_{1-x} \mathrm{Hf}_{x} \mathrm{Fe}_{2}(x=0$ and 0.65$)$, which exhibit reentrant spin-glass freezing. The frequency dependence of ac susceptibility and the bifurcation between zero-field-cooled and field-cooled magnetization curves suggest the complex coexistence of magnetic phases at low temperatures. A long-time relaxation of magnetization is possibly due to the cluster-type and traditional reentrant spin-glass transition, which may be induced by the frustration of long-range $\mathrm{Fe}-\mathrm{Fe}$ interactions. We did not observe any discontinuity indicating long-range magnetic phase transition in the data of specific heat vs temperature, but a broad hump characteristic of spin-glass freezing. The difference between the behaviors of two samples proves the existence of two types of reentrant spin-glass in alloys. The moment arrangements are briefly discussed.

Keywords: reentrant spin-glass; phase separation; alloys

PACS number(s): 75.50.Lk; 75.30.Kz; 75.50.Bb 


\section{Introduction}

Metallic compounds $\mathrm{AFe}_{2}(\mathrm{~A}=\mathrm{Nb}, \mathrm{Hf}$, Ti, and $\mathrm{Sc}$, etc $)$ with the hexagonal C14 Laves phase structure have been of interest to researchers for decades due to their rich physical contents. The compounds of $\mathrm{A}=\mathrm{Hf}$ and $\mathrm{Sc}$ exhibit ferromagnetism with comparatively high Curie temperatures $\left(T_{\mathrm{C}}>500 \mathrm{~K}\right),{ }^{1}$ while stoichiometric $\mathrm{NbFe}_{2}$ was thought to be either paramagnetic (PM) or ferromagnetic (FM) before 1988. ${ }^{2,3}$ However, further nuclear-magnetic-resonance $(\mathrm{NMR})^{4}$ and magnetization measurements ${ }^{5}$ have been interpreted as the presence of spin-density-wave-type (SDW) antiferromagnetic (AFM) order with a Néel temperature $T_{\mathrm{N}} \sim 10 \mathrm{~K}$. The SDW state is very sensitive to stoichiometry and the material becomes FM by slight over doping of either $\mathrm{Nb}$ or $\mathrm{Fe}$, indicating the coexistence of FM and AFM spin fluctuations and the existence of a magnetic quantum critical point (QCP) at or very close to stoichiometric $\mathrm{NbFe}_{2}{ }^{6}$ For a long time, $\mathrm{NbFe}_{2}$ is considered a candidate material for the coexistence of SDW and FM fluctuations because of the close proximity of the reported SDW and ferromagnetism. ${ }^{7,8}$

It is well known that phase coexistence (also named phase separation) is a intrinsic characteristic of perovskite manganites, and the competition between the coexisting FM and charge-order AFM phase of micrometer size can induce frustration and disorder of magnetic exchange interactions, leading to spin-glass (SG) behavior. ${ }^{9-11}$ As a controversial issue, the nonergodic SG state has been found in a wide variety of systems with the following common features: (1) frozen-in magnetic moments below some freezing temperature $T_{f}$ and hence a peak in the frequency-dependent susceptibility, (2) thermo-magnetic irreversibility for frustration and disorder of spins, (3) lack of periodic long-range magnetic order, and (4) remanence and magnetic relaxation on macroscopic time scales below $T_{f}$ when the magnetic field is changed. ${ }^{12,13}$ Furthermore, a certain number of SG systems display reentrant behavior which shares the characteristics of both SG and magnetic orderings. These so-called reentrant spin-glass (RSG) systems undergo a magnetic 
ordering transition and have a spin freezing transition at a lower temperature. ${ }^{14}$ Recently, the observation of slow dynamics resembling (R)SG is also extended to metallic alloys, ${ }^{15-17}$ showing first-order magneto-structural transition.

The glassiness in such a phase-separation system can have two likely origins: first, the slow dynamics of atomic spins due to their metastability as well as disorder capable of pinning the spins, and secondly, the frustration arising from the competing magnetic interactions between two clusters having a distinct magnetic nature. ${ }^{18}$ Mathieu and Tokura $^{19}$ studied the SG-like nonequilibrium dynamics and time relaxation phenomena and proposed that the basic building blocks responsible for the glassy behavior are not really the microscopic atomic spins but rather a macroscopic nanoscale spin cluster or a bigger spin entity which are often referred to as cluster glass or magnetic glass. In this paper, we concentrate on the low-temperature behavior in FM and AFM two-phase mixture $\mathrm{Nb}_{1-x} \mathrm{Hf}_{x} \mathrm{Fe}_{2}$. Magnetization and time relaxation measurements confirm the existence of RSG state at low temperatures. Specific heat is also reported. Our studies suggest that the glassy behaviors are different for $\mathrm{NbFe}_{2}(x=0)$ and the other doped samples $(x \neq 0)$, these phenomena in both samples originate from the frustration of $\mathrm{Fe}-\mathrm{Fe}$ magnetic exchange interactions.

\section{Experimental Details}

Polycrystalline samples of $\mathrm{Nb}_{1-x} \mathrm{Hf}_{x} \mathrm{Fe}_{2}(0 \leq x \leq 0.80)$ were synthesized by an arc melting method using a tungsten electrode under an argon atmosphere. Firstly, the stoichiometric amounts of high purity $\mathrm{Nb}, \mathrm{Hf}$, and $\mathrm{Fe}$ were melted six times for homogeneity on a water-cooled copper hearth. The total weight loss of the sample in this step was less than $0.5 \%$. Then the samples were finally annealed at $1273 \mathrm{~K}$ for one week in evacuated quartz tubes. The crystal structure and phase composition were characterized by x-ray diffraction (XRD) using Rigaku RINT 2250 diffractometer. All samples were confirmed to be of single phase and have the expected hexagonal C14 Laves 
crystal structure, as shown in Fig. 1. As increasing the Hf dopant $x$, the corresponding peak gradually moves to a smaller angle slowly, indicating that the lattice parameters are increasing because of the larger radius of $\mathrm{Hf}$ than $\mathrm{Nb}$. Furthermore, the peak width of $\mathrm{Nb}_{1-x} \mathrm{Hf}_{x} \mathrm{Fe}_{2}$ alloys becomes broadened with substitution of Hf, which indicates that the chemical inhomogeneity becomes rapidly significant. This would induce magnetic inhomogeneity and influence the magnetic behavior in the samples. The magnetization measurements were carried out using a superconducting quantum interference device magnetometer (Quantum Design, MPMS-7) in the temperature range 2 - $300 \mathrm{~K}$, with dc magnetic fields from 0 to $7 \mathrm{~T}$. The ac susceptibility and specific heat measurements were recorded in the temperature range from 2 to $300 \mathrm{~K}$ using a physical property measurement system (PPMS-9) from Quantum Design.

\section{Results and Discussion}

The previous experiments showed that the two sides representative compounds of $\mathrm{NbFe}_{2}$ and $\mathrm{HfFe}_{2}$ carry AFM and FM signs with $T_{\mathrm{N}} \sim 10 \mathrm{~K},{ }^{6} T_{\mathrm{C}} \sim 600 \mathrm{~K},{ }^{20}$ respectively. From the present thermomagnetization $M(T)$ data of $\mathrm{Nb}_{1-x} \mathrm{Hf}_{x} \mathrm{Fe}_{2}$, a clear transition from $\mathrm{AFM}$ of $\mathrm{NbFe}_{2}$ to $\mathrm{FM}$ of $\mathrm{Nb}_{0.20} \mathrm{Hf}_{0.80} \mathrm{Fe}_{2}$ is observed, as shown in Fig. 2, which are collected at $0.1 \mathrm{~T}$ under zero-field-cooled (ZFC) and field-cooled (FC) conditions. The peak in Fig. 2(a) shows the SDW-AFM transition at $T_{\mathrm{N}}$ $\sim 16 \mathrm{~K}$ for $\mathrm{NbFe}_{2}$. A tiny step indicating a weak FM transition at $\sim 230 \mathrm{~K}$ is also observed, which may be caused by the Fe-Fe interaction. Below $T_{\mathrm{N}}$, both $\mathrm{ZFC}$ and FC runs decrease with temperature, but they increase again below $10 \mathrm{~K}$, indicating the existence proof of local short-range FM clusters below $T_{\mathrm{N}}$. The bifurcation between ZFC and FC runs (also called $\lambda$ transition) begins at $\sim 12 \mathrm{~K}$ which is slightly lower than $T_{\mathrm{N}}$ and becomes significant at a lower temperature. This kind of bifurcation is a magnetic characteristic of cluster-type RSG transition. In Fig. 2(b), the FC and ZFC curves are nearly overlapped with each other, both of them are heightened drastically from 50 down to $2 \mathrm{~K}$. It is 
easily to see the magnetization maximum of the sample $x=0.40$ reaches an approximate numerical value with that of $\mathrm{NbFe}_{2}$ at the lowest temperature. The increasing magnetization suggests the increasing FM component with decreasing temperature in $x=0.40$ sample, while $\mathrm{NbFe}_{2}$ has a peak value at $T_{\mathrm{N}} \sim 16 \mathrm{~K}$. With a further increase of $x$, AFM is further suppressed and FM sign is enhanced. In the samples with $0.40<x<0.80$, the FM exchange interaction plays a clear dominant but not absolute role. The Curie temperature $T_{\mathrm{C}}$, defined as the one corresponding to the peak of $d M / d T$ in $M(T)$ data, are 36,125 and $170 \mathrm{~K}$ for $x=0.45,0.65$ and 0.75 , respectively. The bifurcations in Fig.2(c)-(e) clearly show the presence of magnetic inhomogeneity or a glassy transition below $T_{\mathrm{C}}$. As for the sample with $x=0.80$, a pure FM phase is observed and the Curie transition temperature $T_{\mathrm{C}}$ is evidenced to be around room temperature.

The $M(T)$ irreversible behavior below $T_{\mathrm{N}}$ of $\mathrm{NbFe}_{2}$ implies some $\mathrm{FM}$ contribution to magnetization or a glassy transition where spins would be frozen randomly below $T_{f}$, and it is necessary to combine other experiments to clarify it. The temperature dependence of ac magnetization for $\mathrm{NbFe}_{2}$ is measured in the temperature range of $2-300 \mathrm{~K}$, the real part $M^{\prime}(T)$ with variable frequency $f$ around the phase transition temperature is shown in Fig. 3. The peak in $M^{\prime}(T)$ exhibits obvious frequency dependence in an ac magnetic field $H_{\mathrm{ac}}=10$ Oe. As $f$ increasing, the peak position shifts to a higher temperature whereas the magnitude decreases, indicating typical SG behavior. It is interesting that Curie-Weiss behavior is absent for the ac magnetization data in the whole temperature range 2-300 $\mathrm{K}$ (not shown here). This might suggest the presence of inhomogeneous short-range FM correlations below $\sim 230 \mathrm{~K}$ and the AFM phase transition at $T_{\mathrm{N}} \sim 16$ $\mathrm{K}$. What we are interest in is the lower-temperature behavior, such as the minimum of $M^{\prime}$ around 10 $\mathrm{K}$. Although AFM interaction is dominant below $T_{\mathrm{N}}, \mathrm{FM}$ clusters partly coexist with the AFM phase toward phase separation. The increasing $M^{\prime}$ and $M$ indicate the growing FM clusters with decreasing temperature from $10 \mathrm{~K}$, which is a character of cluster glass. The inset of Fig. 3 shows the time 
dependence of magnetization $M(t)$, measured after ZFC process from room temperature to the desired measurement temperatures and applying the field $H=0.1 \mathrm{~T}$. After the field is applied (time $=$ 0), the magnetization at $4.2 \mathrm{~K}$ (below $T_{f}$ ) exhibits a slow nonexponential increase, indicative of a nonequilibrium nature. The $M(t)$ data collected in the ZFC mode at $4.2 \mathrm{~K}$ show a $4 \%$ change in $10^{4} \mathrm{~s}$, while at $16 \mathrm{~K}$ (above $T_{f}$ ) the change is only $0.1 \%$. This long time relaxation of magnetization is another signature of glassiness. Because of the FM and AFM transition above the bifurcation of ZFC and $\mathrm{FC}$ runs, it is suitable to term this behavior in $\mathrm{NbFe}_{2}$ cluster-type RSG, which shares the characteristics of both SG and magnetic ordering. As for the samples with $0.40<x<0.80$, the competition between the dominant FM and the local AFM phase associated with the significant magnetic and chemical inhomogeneity are probably the main reasons to produce disordered glassy state. Considering of the different magnetic ground states for these two series, we chose the two particular samples with $x=0$ and 0.65 for the following studies.

In order to detect the existence of short-range FM or AFM correlation in the magnetic disorder system, the magnetization loops for $x=0$ and 0.65 are given in Fig. 4(a). At $300 \mathrm{~K}$, the $M-H$ loops for both samples are almost linear and there is no hysteresis. However, a foreseeable S-shape $M-H$ loop can be observed at $4.2 \mathrm{~K}$, which is a typical behavior of SG systems. ${ }^{21}$ The AFM ground state for $\mathrm{NbFe}_{2}$ is confirmed when the applied magnetic field reaches $5 \mathrm{~T}$. On the other hand, the sample with $x=0.65$ exhibits a FM ground state at zero field at $4.2 \mathrm{~K}$. It is easy to understand that the FM exchange interaction is dominant below $T_{\mathrm{C}}(\sim 125 \mathrm{~K}$ for present sample) for a ferromagnet. However, the rising magnetization with field and small traces fully explain the existence of magnetic inhomogeneity which may be induced by the frustration of FM interaction. As shown in Fig. 4(b) and (c) the Arrott plots for both samples at $T=4.2 \mathrm{~K}$ are also displayed. Consistent with the above discussion, the Arrott plot for sample $x=0.65$ shows small traces (not shown for clear), and the finite $M^{2}$ intercept for zero $H / M$ is a clear indication of a remanent magnetization and hence of 
ferromagnetism. ${ }^{6}$ For no doped sample $\mathrm{NbFe}_{2}, x=0$, the Arrott plot suggests a AFM ground state and the negative slope can be associated with the S-shape $M-H$ loop as shown in Fig. 3(a),

For comparison, Fig. 5 gives the temperature and frequency dependence of ac magnetization of the sample $x=0.65$. In Fig. 5(a), the real part $M^{\prime}$ of ac magnetization exhibits an additional clear hump at a temperature much below $T_{\mathrm{C}} \sim 125 \mathrm{~K}$. The hump decreases and moves to a higher temperature with increasing the frequency as indicated by the arrow. This is also a typical characteristic of RSG. Different from the former sample, which is considered as cluster-type RSG, the most interesting characteristic of this sample is the rapid monotonous decrease of $M^{\prime}$ while temperature goes down from $T_{f}$ to $2 \mathrm{~K}$, evidently displaying the intrinsic frustration of FM spins and the buildup of a disordered glassy magnetic phase. That is to say, the magnetic behavior of $x=0.65$ sample below $T_{f}$ indicates the freezing of traditional glassy disorder for atomic spins. Fig. $5(\mathrm{~b})$ presents the imaginary part $M^{\prime \prime}$ versus temperature curves. A sharp peak, corresponding to the hump in Fig. 5(a), remarks the absorption of energy and the spin freezing temperature $T_{f} . T_{f}$ as a function of frequency is also displayed in the inset. By fitting the frequency dependence of the peak shift using $K=\triangle T_{f} /\left(T_{f} \triangle \log f\right)$, we obtained $K=0.093$. This is very close to the values found in some other SG systems. ${ }^{23}$ In addition, this kind of RSG often includes FM clusters, which can be proven by the spontaneous magnetization and nonzero $M^{2}$ intercept at zero field at $4.2 \mathrm{~K}$ for $x=0.65$ as shown in Fig. 4 (a) and (b), respectively. ${ }^{6}$

For more information about the ground-state properties at low temperatures, we carried out the measurement of specific heat vs temperature $C(T)$ plotted in fig. 6(a) and (b), for $x=0$ and $x=0.65$ respectively. No discontinuity indicating long-range order is observed for neither of the samples around the temperature at which $M(T)$ or $M^{\prime}(T)$ has peak-type (at $T_{\mathrm{N}}$ ) and irreversibility-type (at $T_{f}$ ) anomalies. The lack of a $C(T)$ peak is exactly one of the features for SG systems, which may be owed to the much released entropy above $T_{f .}{ }^{22,23}$ 
At low temperatures, the specific heat data can be fitted using $C=\gamma_{\mathrm{SG}} T+\beta T^{3}$ [insets of Fig. 6(a) and (b)], where $\gamma_{\mathrm{SG}} T$ and $\beta T^{3}$ correspond to the electronic and phonon contribution, respectively. The variable Sommerfeld coefficient $\gamma_{\mathrm{SG}}$ is commonly found in SG systems, implying a constant density of states of the low-temperature magnetic excitations. ${ }^{24-26}$ The obtained $\gamma_{\mathrm{SG}}$ is $13.6 \mathrm{~mJ} /\left(\mathrm{mol} . \mathrm{K}^{2}\right)$ for $\mathrm{NbFe}_{2}$, which agrees very well with previous results ${ }^{6}$ and that predicted by band-structure calculations; ${ }^{27,28}$ and $\gamma_{\mathrm{SG}}=42.6 \mathrm{~mJ} /\left(\mathrm{mol}_{\mathrm{K}} \mathrm{K}^{2}\right)$ for $\mathrm{Nb}_{0.35} \mathrm{Hf}_{0.65} \mathrm{Fe}_{2}$. The Debye temperature $\Theta_{\mathrm{D}}$ can be calculated from $\beta$ through $\Theta_{\mathrm{D}}=\left(12 \pi^{4} N \mathrm{R} / 5 \beta\right)^{1 / 3}$ to be $\Theta_{\mathrm{D}}=342.9 \mathrm{~K}$ for $\mathrm{NbFe}_{2}$ and $319.3 \mathrm{~K}$ for $\mathrm{Nb}_{0.35} \mathrm{Hf}_{0.65} \mathrm{Fe}_{2}$, where $N$ is the atomic number in the chemical formula $(N=3$ for present system) and $\mathrm{R}$ is the gas constant. It should be noted that the clear extra contribution beyond the electronic and phonon contributions at low temperatures, which could well be attributed to the local-moment fluctuations as is also found in the Fe-based SG superconductors. ${ }^{29}$ In addition, as opposed to the usual $\lambda$-shape anomaly, there is a broad platform of $C / T$ around $T_{\mathrm{N}} \sim 16 \mathrm{~K}$ for $\mathrm{NbFe}_{2}$, as indicated by the arrow in the inset of Fig. 6(a). The arrow in the inset of Fig. 6(b) marks the temperature at which $C(T)$ deviates from $C / T=\gamma_{\mathrm{SG}}+\beta T^{2}$, in accord with the observed anomaly of ac magnetization with onset at about twenties of Kelvin.

Studies of the doping evolution of the phase diagram have shown that this SDW-AFM state is very sensitive to changes in stoichiometry. ${ }^{6}$ Dilute over doping of either $\mathrm{Nb}(y<0)$ or $\mathrm{Fe}(y>0)$ in $\mathrm{Nb}_{1-y} \mathrm{Fe}_{2+y}$ would suppress the SDW-AFM phase and make the material FM. Then a mixed phase of SDW and FM order is formed in the range of $0 \leq y \leq 0.02$. Compared with $\mathrm{Nb}_{0.993} \mathrm{Fe}_{2.007}(y=0.007)$ (FIG. 14 in Ref. 6), which has a very similar ac magnetization curve and approximate transition temperature $T_{\mathrm{N}} \sim 18 \mathrm{~K}$ with present $x=0$ sample (see Fig. 3), one can find the present sample of nominal $\mathrm{NbFe}_{2}$ should be refined to be $\mathrm{Nb}_{1-y} \mathrm{Fe}_{2+y}(0<y<0.007)$ which is located in the phase diagram around the point of crossover between the pure FM phase and the SDW one. The small deviation might be caused by the dilute weight loss during sample preparation, which would 
introduce defects and disorder in the sample. Clearly, the AFM transition of present sample appears at $\sim 16 \mathrm{~K}$, slightly higher than the former reported $T_{\mathrm{N}} \sim 10 \mathrm{~K}$. A weak FM transition is also observed at $\sim 230 \mathrm{~K}$ as shown in Fig. 2(a). These complex interactions, such as SDW-AFM, short-range FM correlations, and disorder induced by magnetic inhomogeneity and defects, lead to a complex coexistent phase and the frustration of competing magnetic interactions, which may be the origination of cluster-type RSG at lower temperatures.

As $x$ increasing from zero, a new $\mathrm{FM} \mathrm{HfFe} 2$ phase is introduced into the sample, the chemical inhomogeneity becomes significant. With the FM component increasing, the magnetization is enhanced, the AFM phase is suppressed and finally vanished in $x=0.80$ sample. Therefore, the competing magnetic phases associated with the magnetic and chemical inhomogeneity result in the disorder and frustration of FM spins in $0.40<x<0.80$ samples. When the temperature dropped below $T_{f}$, randomly arranged atomic spins would be frozen-in and can not follow with the magnetic field rotation freely. Then significant frequency-dependent effect is observed in ac susceptibility curves. Furthermore, the lower the temperature is, the more atomic spins are frozen-in, which results in the monotone reduction of magnetization as temperature decreasing from $T_{f}$ to $2 \mathrm{~K}$. This is a typical traditional RSG behavior.

A common feature to this kind of $\mathrm{AFe}_{2}$ materials is that magnetic $\mathrm{Fe}$ atoms occupy two sites in C14 Laves structure, $2 a$ and $6 h$, which may behave differently from a magnetic point of view, while the A-site atoms ( $\mathrm{Nb}$ and $\mathrm{Hf}$ ) have a very small moment that can be ignored. The crystal structure of C14 Laves phase $\mathrm{AFe}_{2}$ compounds is shown in Fig. 7. The exchange interaction between $\mathrm{Fe}(6 h)$ atoms is always FM within the same $6 h$ layer. The interaction between the adjacent $\mathrm{Fe}(6 h)$ layers is governed by the $\mathrm{A}$-site atom. Take $\mathrm{TiFe}_{2}$ as an example, where $\mathrm{Ti}$ has a approximate covalent radius with $\mathrm{Nb}$ and so one may conjecture that $\mathrm{NbFe}_{2}$ is similar to $\mathrm{TiFe}_{2}$, only $3 / 4$ of the $\mathrm{Fe}$ atoms are involved in the AFM structure, namely those occupying $6 h$ sites, while there is no moment on the 
$\mathrm{Fe}(2 a)$ sites. The ordered spin arrangement is made up of layers of ferromagnetically aligned $6 h$ spins with opposite orientations on adjacent layers. Since $2 a$ site is an inversion center for $6 h$ sublattice (see Fig. 7), the net exchange field experienced by $2 a$ spins vanishes for symmetry. As a consequence, $\mathrm{Fe}(2 a)$ atoms exhibit null hyperfine fields or null magnetic moments in Mössbauer and neutron diffraction experiments, respectively. ${ }^{30}$ However, in the case of the $\mathrm{Nb}_{1-x} \mathrm{Hf}_{x} \mathrm{Fe}_{2}$ alloys, the competition between FM and AFM interactions indicates that the $2 a$ site may play an important role in the magnetism even though it may not have ordered moments, because of the unsearchable SDW order for $\mathrm{NbFe}_{2}$ and the FM arrangement of both $6 h$ and $2 a$ for $\mathrm{HfFe}_{2}$ [see Fig. 8(a)]. The origin of the frustration is noncollinearity of the $\mathrm{Fe}(6 h)$ magnetic sublattice. That is, as indicated in Fig. 8(b), as the moments in the adjacent $\mathrm{Fe}(6 h)$ layers tilt in opposite directions, the $\mathrm{FM} \mathrm{Fe}(2 a)-\mathrm{Fe}(6 h)$ exchange bonds tend to be frustrated in the sense that they are not energetically satisfied. ${ }^{31}$ Magnetic frustration is a familiar feature in the lattice in which magnetic atoms form a network of vertex-sharing tetrahedra.

Rechenberg et $a l^{32}$ reported that there are strong magnetic interactions between $\mathrm{Fe} 2 a$ and $6 h$ layers. The interlayer $2 a-2 a$ and $6 h-6 h$ interactions are also strong, which implies competing magnetic interactions. Furthermore, the electronic structure and magnetic interactions are three dimensional (3D). This implies that geometric frustration based on AFM interactions in the 2D Kagome planes is unlikely to be the main player in the quantum criticality. Based on these earlier results, we suggest that the low-temperature magnetic order in stoichiometric $\mathrm{NbFe}_{2}$ may take the form of a SDW with a long-wavelength helical state, Fe on the $6 h$ sites form FM sheets, which are stacked antiferromagnetically along $c$-axis, as predicted in many other works. ${ }^{6,33}$

\section{Summary}

We studied the $\mathrm{dc}$ and ac magnetization and specific heat properties of $\mathrm{Nb}_{1-x} \mathrm{Hf}_{x} \mathrm{Fe}_{2}$ alloys, 
distinguishable glassy magnetic phases are observed with variable dopant $x$. In nominal stoichiometric $\mathrm{NbFe}_{2}, x=0$, a weak FM transition at $\sim 230 \mathrm{~K}$ and a clear AFM transition at $\sim 16 \mathrm{~K}$ are both observed, the frequency dependence of ac magnetization and a long time relaxation effect suggest the cluster-type RSG freezing, which can be ascribed to the presence of competing phase separation and disorder induced by defects. On the other hand, in $x=0.65$ sample, FM interaction is dominant after the collapse of long-range AFM order, the competition between FM and the coexisting residual canted AFM interactions associated with the significant magnetic and chemical inhomogeneity may be the main reasons to produce the traditional RSG transition. Furthermore, the specific heat data for both samples also show the spin fluctuation contribution below the spin-freezing temperature. The complex magnetic phase separation behavior at low temperatures in both samples leads to the frustration of long-range $\mathrm{Fe}-\mathrm{Fe}$ interactions, which should be the origin of the cluster and traditional RSG transition. 


\section{Reference}

${ }^{1}$ B. Ya. Kotur, E. Gratz, E. Bauer, G. Hilscher, A. Kottar, H. Michor, Ch. Reichl, G. Wiesinger and A. S. Markosyan, Journal of Alloys and Compounds 278, 49-59 (1998).

${ }^{2}$ M. Nevitt, C. Kimball, and R. Preston, Proceedings of the International Conference on Magnetism, Nottingham, 1964 (The Institute of Physics and The Physical Society, London, 1964), Vol. 169, p137.

${ }^{3}$ Y. Yamada, Y. Kitaoka, K. Asayama, and A. Sakata, J. Phys. Soc. Jpn. 53, 3198 (1984).

${ }^{4}$ Y. Yamada, and A. Sakata, J. Phys. Soc. Jpn. 57, 46 (1988).

${ }^{5}$ Y. Yamada, R. Moru, K. Hirano, T. Hirotsuji, G. Obara, and T. Nakamura, J. Magn. Magn. Mater. 310, 1812 (2007).

${ }^{6}$ M. Brando, W. J. Duncan, D. Moroni-Klementowicz, C. Albrecht, D. Grüner, R. Ballou, and F. M. Grosche, Phys. Rev. Lett. 101, 026401 (2008); D. Moroni-Klementowicz, M. Brando, C. Albrecht, W. J. Duncan, and F. M. Grosche, D. Grüner and G. Kreiner, Phys. Rev. B 79, 224410 (2009).

${ }^{7}$ M. R. Crook and R. Cywinski, J. Magn. Magn. Mater. 140-144, 71 (1995).

${ }^{8}$ M. Shiga and Y. Nakamura, J. Phys. Soc. Jpn. 56, 4040 (1987).

${ }^{9}$ Shipeng Wang, Jincang Zhang, Guixin Cao, Chao Jing, and Shixun Cao, Phys. Rev. B 76, 054415 (2007).

${ }^{10}$ F. Rivadulla, M. A. López-Quintela, and J. Rivas, Phys. Rev. Lett. 93, 167206 (2004).

${ }^{11}$ E. Dagotto, New J. Phys. 7, 67 (2005).

${ }^{12}$ N. Hanasaki, K. Watanabe, T. Ohtsuka, I. Kézsmárki, S. Iguchi, S. Miyasaka, and Y. Tokura, Phys. Rev. Lett. 99, 086401 (2007).

${ }^{13}$ Kim Myung-Whun, J. S. Kim, T. Katsufuji, and R. K. Kremer, Phys. Rev. B 83, 024403 (2011).

${ }^{14}$ M. Viswanathan and P. S. Anil Kumar, Phys. Rev. B 80, 012410 (2009).

${ }^{15}$ Yongsheng Liu, Jincang Zhang, Shixun Cao, and Zhongming Ren, J. Appl. Phys. 104, 043901 (2008).

${ }^{16}$ M. K. Chattopadhyay, S. B. Roy, and P. Chaddah, Phys. Rev. B 72, 180401(R) (2005).

${ }^{17}$ S. B. Roy, M. K. Chattopadhyay, P. Chaddah, J. D. Moore, G. K. Perkins, L. F. Cohen, K. A. Gschneidner Jr., and V. K. Pecharsky, Phys. Rev. B 74, 012403 (2006).

${ }^{18}$ A. Bhattacharyya, S. Giri, and S. Majumdar, Phys. Rev. B 83, 134427 (2011).

${ }^{19}$ R. Mathieu and Y. Tokura, J. Phys. Soc. Jpn. 76, 124706 (2007).

${ }^{20}$ T. Nakamichi, K. Kai, Y. Aoki, K. Ikeda, M. Yamamoto, J. Phys. Soc. Japan 29, 794 (1970).

${ }^{21}$ J. J. Ying, A. F. Wang, Z. J. Xiang, X. G. Luo, R. H. Liu, X. F. Wang, Y. J. Yan, M. Zhang, G. J. Ye, P. Cheng, and X. H. Chen, e-print arXiv:1012.2929.

${ }^{22}$ Masakazu Ito, Toru Hisamatsu, Tsugumi Rokkaku, Iduru Shigeta, Hirotaka Manaka, Norio Terada, and Masahiko Hiroi, Phys. Rev. B 82, 024406 (2010).

${ }^{23}$ Hechang Lei, Milinda Abeykoon, Emil S. Bozin, and C. Petrovic, Phys. Rev. B 83, 180503(R) 
(2011).

${ }^{24}$ C. Y. Huang, J. Magn. Magn. Mater. 51, 1 (1985).

${ }^{25}$ D. Meschede, F. Steglich,W. Felsch, H.Maletta, andW. Zinn, Phys. Rev. Lett. 44, 102 (1980).

${ }^{26}$ N. P. Raju, E. Gmelin, and R. K. Kremer, Phys. Rev. B 46, 5405 (1992).

${ }^{27}$ N. Takayama, and M. Shimizu, J. Phys. F 18, L83 (1988).

${ }^{28}$ J. Inoue and M. Shimizu, J. Magn. Magn. Mater. 79, 265 (1989).

${ }^{29}$ F. Bondino, E. Magnano, M. Malvestuto, F. Parmigiani, M. A. McGuire, A. S. Sefat, B. C. Sales, R. Jin, D. Mandrus, E. W. Plummer, D. J. Singh, and N. Mannella, Phys. Rev. Lett. 101, 267001 (2008).

${ }^{30}$ H. R. Rechenberg, L. Morellon, P. A. Algarabel, and M. R. Ibarra, Phys. Rev. B 71, 104412 (2011).

${ }^{31}$ N. N. Delyagin, A. L. Erzinkyan, V. P. Parfenova, I. N. Rozantsev, G. K. Ryasny, J. Magn. Magn. Mater. 320, 1853 (2008).

${ }^{32}$ Alaska Subedi, and David J. Singh, Phys. Rev. B 81, 024422 (2010).

${ }^{33}$ D. A. Tompsett, R. J. Needs, F. M. Grosche, and G. G. Lonzarich, Phys. Rev. B 82, 155137 (2010). 


\section{Figure Captions}

Fig. $1 \mathrm{X}$-ray-diffraction patterns at room temperature for $\mathrm{Nb}_{1-x} \mathrm{Hf}_{x} \mathrm{Fe}_{2}$. The patterns are shifted downwards from top $(x=0)$ to bottom $(x=0.80)$ for the sake of comparison.

Fig. 2 Temperature dependence of magnetization under an external magnetic field $H=0.1 \mathrm{~T}$ from 2 to $300 \mathrm{~K}$ for $\mathrm{Nb}_{1-x} \mathrm{Hf}_{x} \mathrm{Fe}_{2}$ plotted by logarithmic $x$-axis scale, for the visual of the divarication of ZFC and FC curves at lower temperatures.

Fig. 3 (Color online) Temperature dependence of ac susceptibility for $\mathrm{NbFe}_{2}$ at an ac field $H_{\mathrm{ac}}=$ 10 Oe with different frequencies. The figure inset shows the time dependence of ZFC magnetization of $\mathrm{NbFe}_{2}$, with $H=0.1 \mathrm{~T}$.

Fig. 4 (Color online) (a) Magnetization curves of $\mathrm{Nb}_{1-x} \mathrm{Hf}_{x} \mathrm{Fe}_{2}$ measured at $4.2 \mathrm{~K}$ and $300 \mathrm{~K}$ up to $5 \mathrm{~T}$. And the Arrot plot at $T=4.2 \mathrm{~K}$ for (b) $x=0.65$ and (c) $x=0$.

Fig. 5 (Color online) Temperature dependence of ac susceptibility for $\mathrm{Nb}_{1-x} \mathrm{Hf}_{x} \mathrm{Fe}_{2}(x=0.65)$ at an ac field $H_{\mathrm{ac}}=10$ Oe with different frequencies, (a) the real part and (b) the imaginary part. The arrow marks the direction of movement of the hump. The inset shows frequency dependence of the spin freezing temperature $T_{f}$. The solid line is the linear fit to the $T_{f}$ data.

Fig. 6 (Color online) Temperature dependence of specific heat for (a) $x=0$, and (b) $x=0.65$. The figure insets show low-temperature specific-heat data in the plot of $C / T$ vs $T^{2}$. The solid line is the fitting curve using the formula $C / T=\gamma_{\mathrm{SG}}+\beta T^{2}$. The arrows mark the approximate location of $T_{\mathrm{N}}$ and $T_{f}$ for two samples, respectively.

Fig. 7 Crystal structure of $\mathrm{C} 14$ Laves phase $\mathrm{AFe}_{2}$ compounds. The atom number ratio $\mathrm{Fe}(6 h): \mathrm{Fe}(2 a)=3: 1$. The arrow marks the direction of $c$-axis.

Fig. 8 (Color online) Schematic diagram of the spin configuration for (a) the FM arrangement of $\mathrm{Fe}$ spins and (b) the frustration of $\mathrm{Fe}(2 a)$. Only Fe atoms are shown and the $2 a$ and $6 h$ rows are as indicated. The arrow marks the direction of $c$-axis. 
Fig. 1

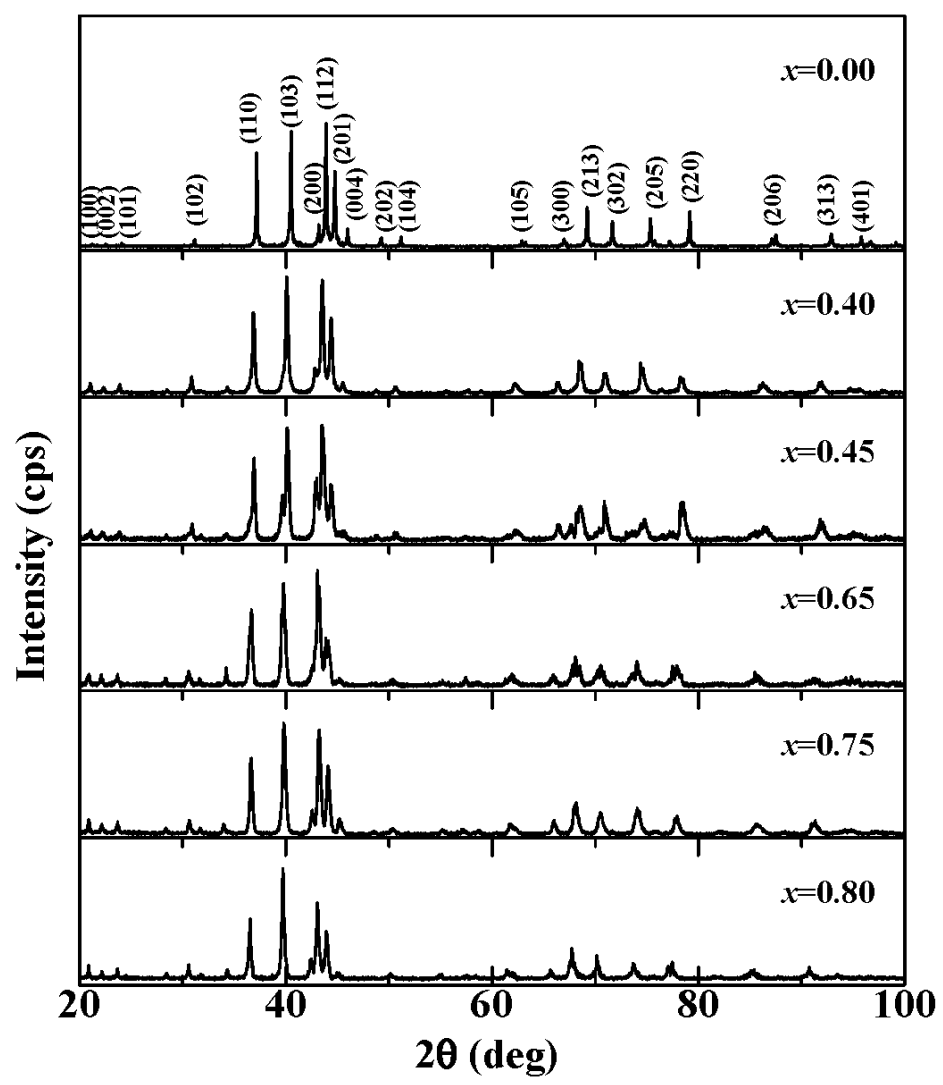


Fig. 2

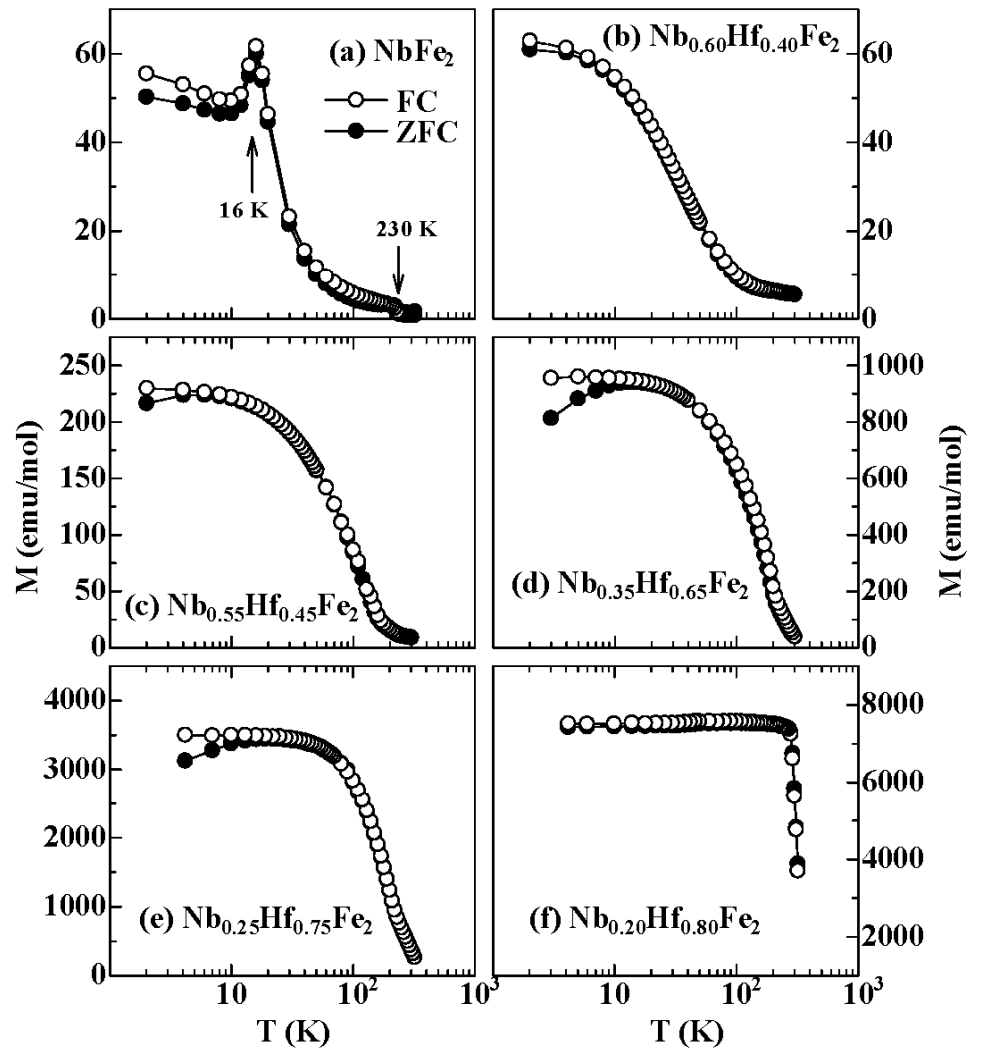


Fig. 3

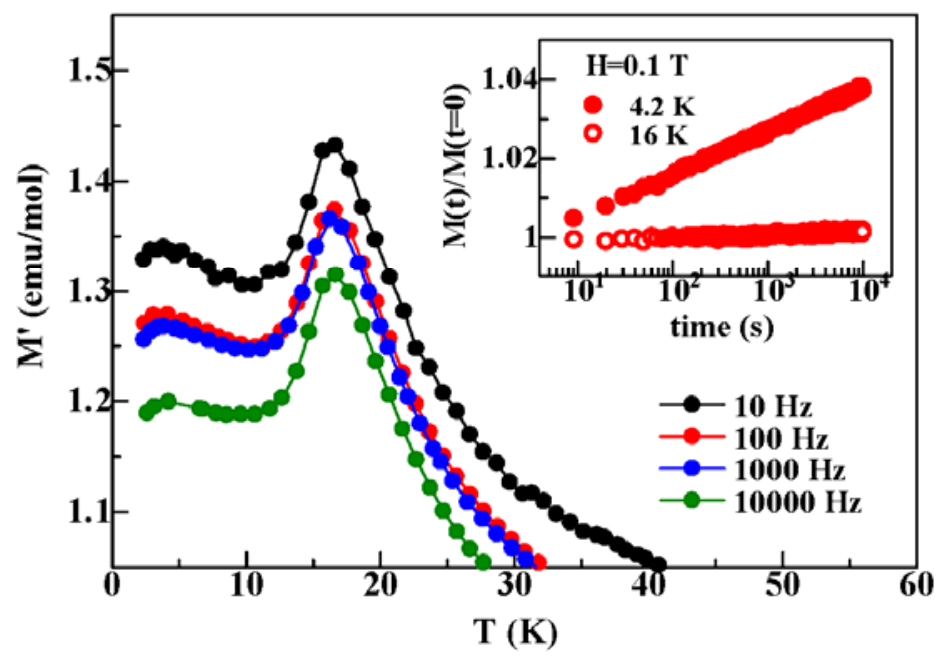


Fig. 4

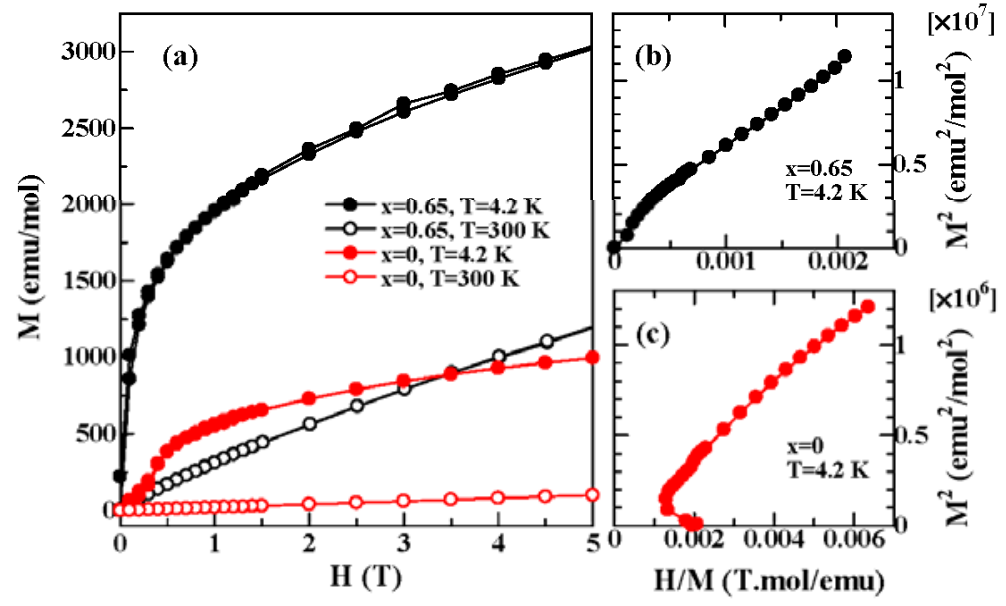


Fig. 5

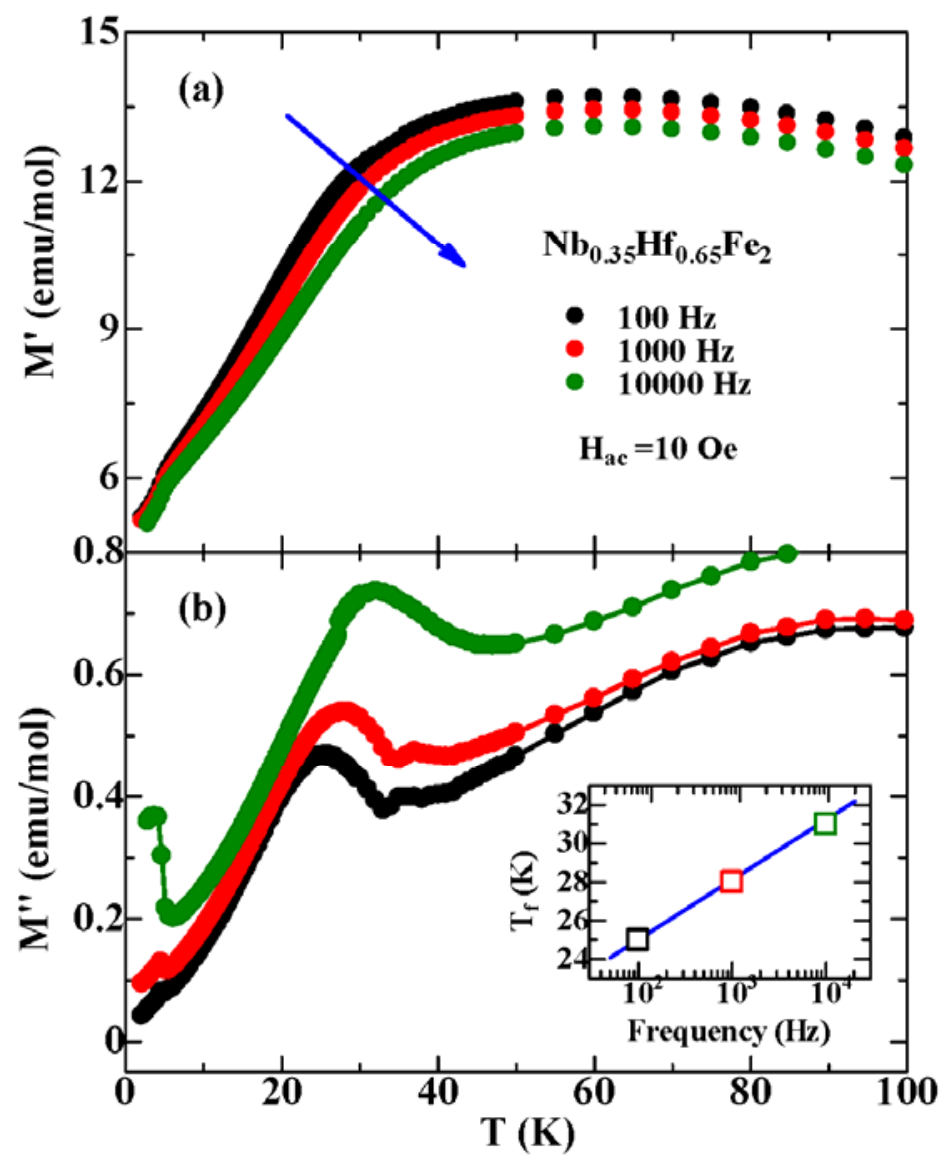



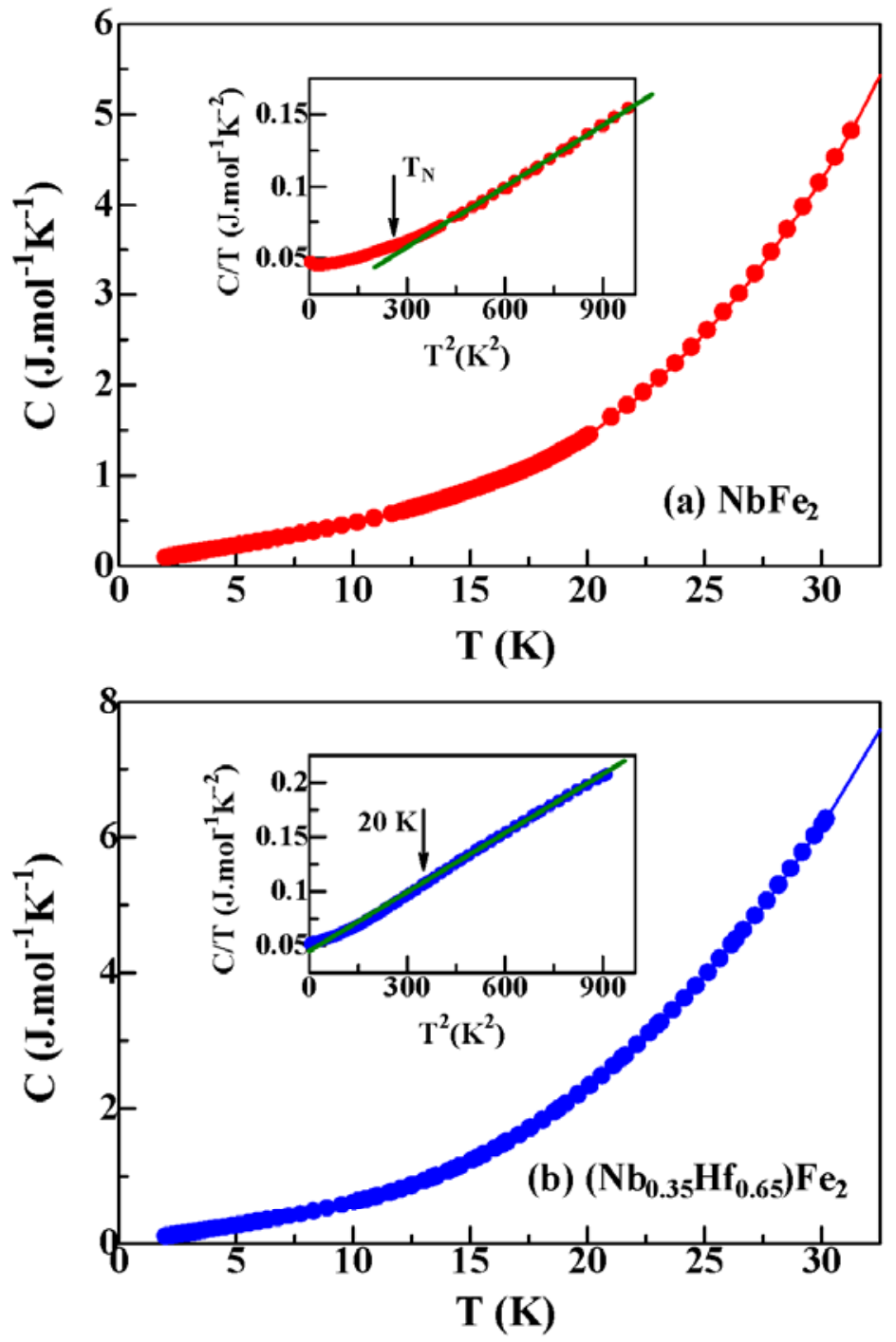
Fig. 7

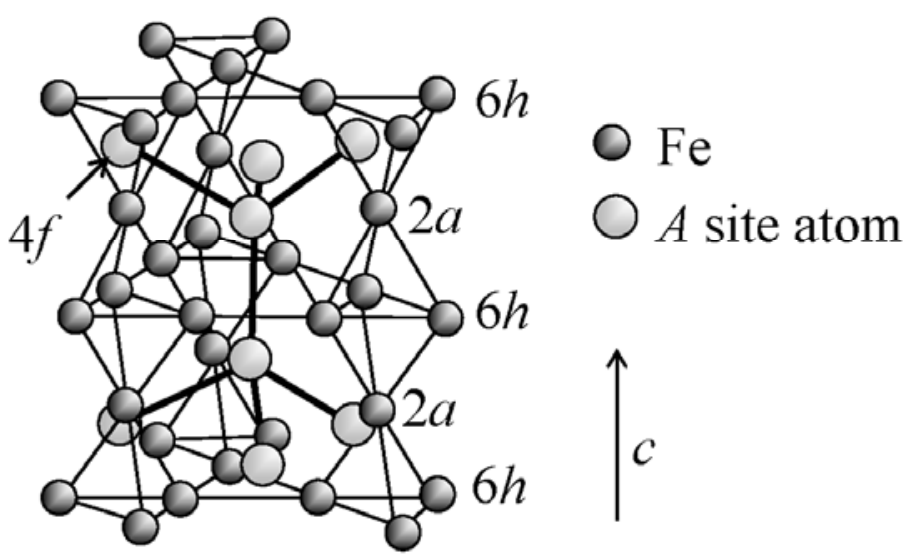




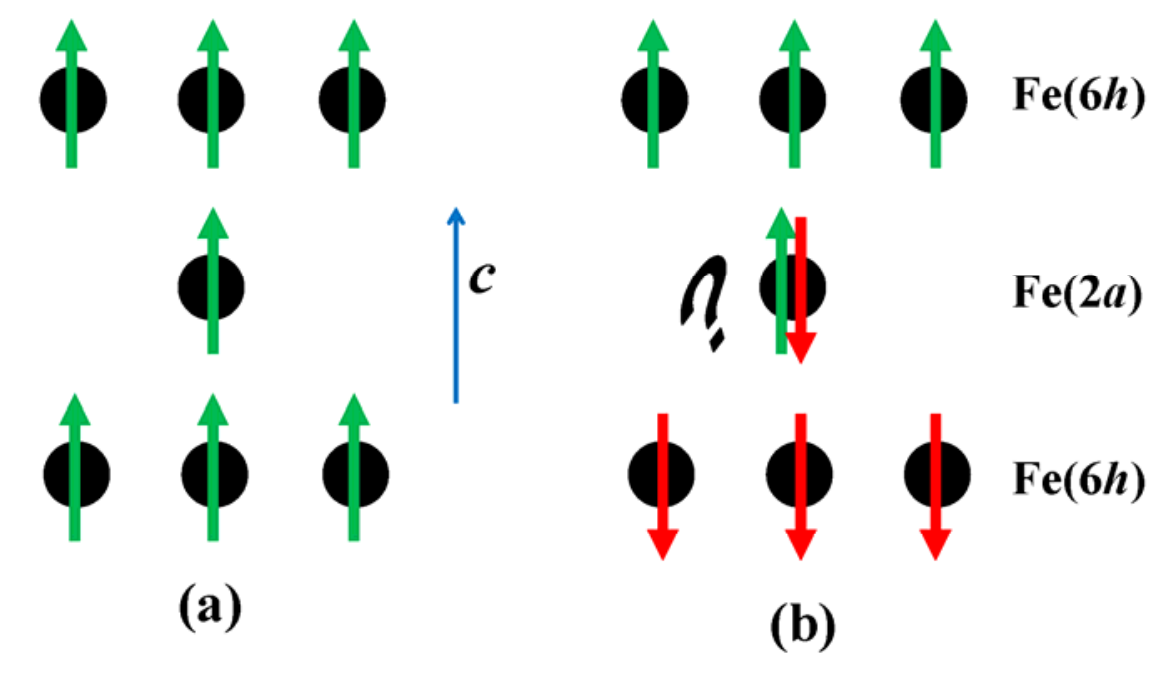

